\title{
Refractive Indices of Poly[(2,5-didecyloxy-1,4-phenylene)-alt-(2,5-thienylene)] Polymer
}

\author{
${ }^{1}$ Asim MANTARCI* and ${ }^{2}$ Bayram GÜNDÜZ \\ *1Department of Medical services and Techniques, Muş Alparslan University, Turkey \\ ${ }^{2}$ Department of Science Education, Muş Alparslan University, Muş, Turkey
}

\begin{abstract}
In this study, optical characteristics of poly[(2,5-didecyloxy-1,4-phenylene)-alt-(2,5thienylene)] (PODT) polymer were researched by using solution processed method. The refractive indices of this polymer for various physical models (Moss, Ravindra, Herve-Vandamme, Reddy and Kumar-Singh) were calculated and the refractive index dispersion of the PODT polymer was analyzed in detail. It was achieved that the refractive index of the PODT varies from 2.462 to 2.889 (Herve-Vandamme model is the lowest one, Reddy model is the highest one). Furthermore, the reflectance of the polymer is greater at wavelengths less than about $500 \mathrm{~nm}$, which is very applicable for devices that require high reflective properties. It was concluded that achieved results can be significant for organic devices that require specific reflectance and refractive index properties.
\end{abstract}

Key words: Herve-Vandamme model, Refractive index, optical analysis, Reflective property

\section{Introduction}

Conjugated polymers have attracted an increasing amount of attention in recent years for various organic electronic devices due to their potential advantages over inorganic and small-molecule organic semiconductors [1]. In the literature, there has been a need to detailed understanding of refractive indices dispersion of and some optical parameters of this material for developing better organic devices.

In here, we studied the optical characteristics of poly[(2,5-didecyloxy-1,4-phenylene)-alt-(2,5thienylene)] (PODT) polymer. Also, according to several physical models, the refractive index dispersion of the PODT polymer was found and analyzed in detail.

\section{Materials and Method}

As a solvent, Chloroform $(\mathrm{CHCl} 3)$ was processed. As researched material, poly[(2,5-didecyloxy1,4-phenylene)-alt-(2,5-thienylene)] (PODT) was used in this work. They were both bought from corporation of Sigma-Aldrich. The chemical structure of the PODT polymer is shown in Fig. 1. 


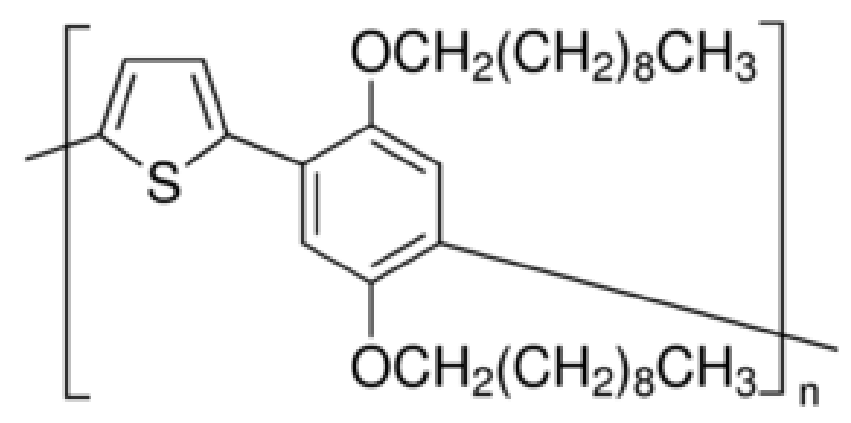

Fig. 1 The chemical structure of the PODT polymer

The material was weighed with an analytical balance and dissolved with stirring in the solvent. Obtained polymers were measured by absorbance spectrometry at room temperature. Moss, Ravindra, Herve-Vandamme, Reddy and Kumar-Singhn physical models were used to have refractive index dispersion parameters of the polymer. All results were analyzed in detail.

\section{Results}

Reflectance is the fraction of the total radiant flux incident upon a surface that is reflected and that varies according to the wavelength distribution of the incident radiation [2]. We took the reflectance spectra of the PODT polymer as seen in Fig. 2.

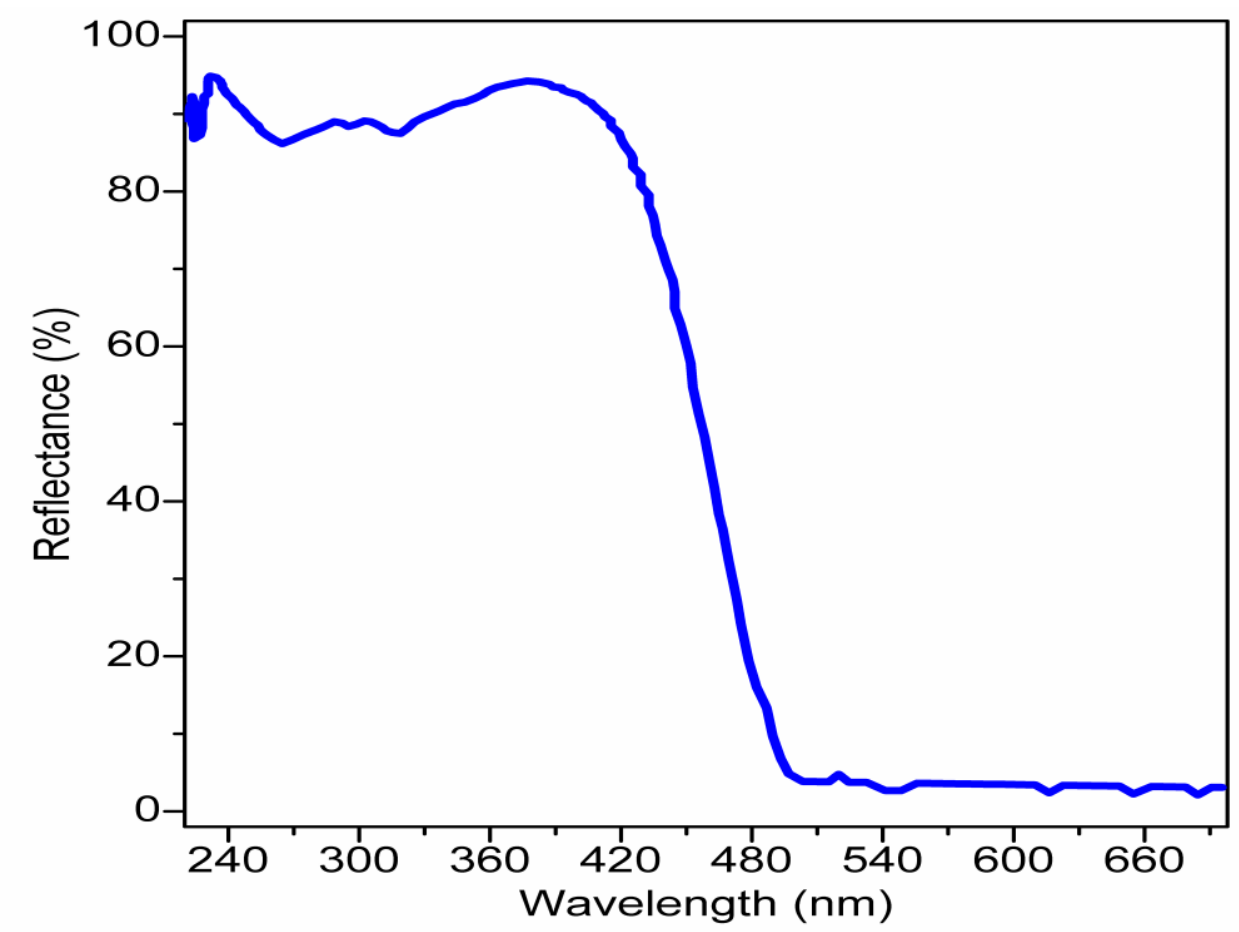

Fig. 2 The reflectance spectra of the PODT polymer 
The reflectance of the PODT polymer is greater at wavelengths less than about $500 \mathrm{~nm}$. Particularly at wavelengths where reflectance is greater than $50 \%$, the PODT polymer is crucial for materials that require high reflective properties.

The PODT polymer showed the direct optical band gap $\left(\mathrm{E}_{\mathrm{gd}}\right)$ behavior and the $\mathrm{E}_{\mathrm{gd}}$ value of the PODT polymer was found to be $2.575 \mathrm{eV}$.

The refractive index ( $\mathrm{n}$ ) is an important parameter in optical science and engineering. There are many physical models including Moss, Ravindra, Herve-Vandamme, Reddy and KumarSingh based on optical band gap [3-9]. We obtained refractive indices of the PODT polymer and plotted in Fig. 3. As seen in Fig. 3, the refractive index (2.462) of the PODT for HerveVandamme relation is the lowest one, while the refractive index (2.889) of the PODT for Reddy relation is the highest one.

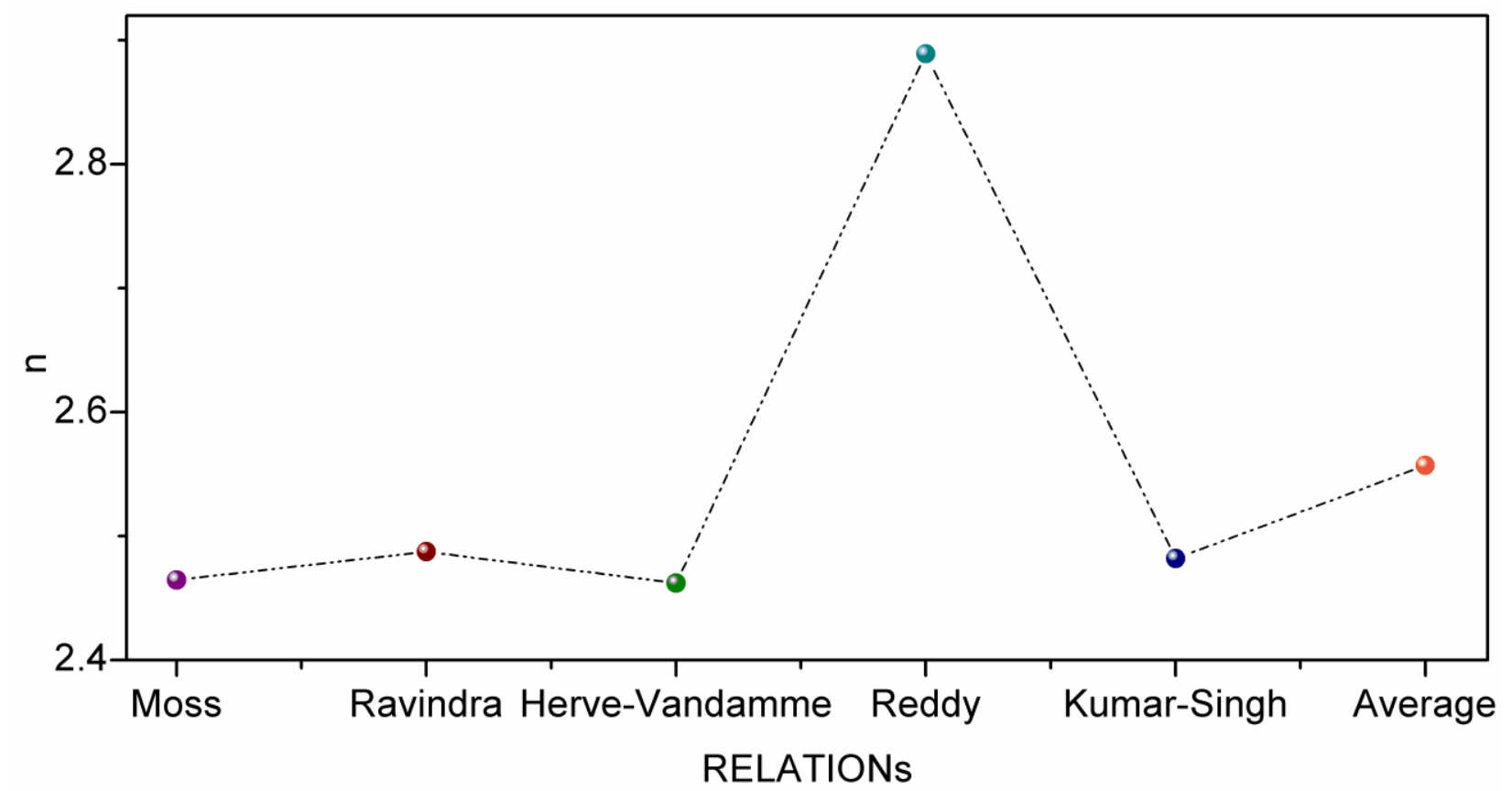

Fig. 3 The refractive indices of the PODT polymer for various relations

\section{Conclusions}

Based on many different physical model (Moss, Ravindra, Herve-Vandamme, Reddy and KumarSingh), the refractive indices of the PODT polymer were found and the results were compared with each other. The PODT polymer exhibited the direct optical band gap $\left(\mathrm{E}_{\mathrm{gd}}\right)$ behavior, which can be very significant for optoelectronic applications. The $\mathrm{E}_{\mathrm{gd}}$ value of the PODT polymer was 
$2.575 \mathrm{eV}$. The refractive index of the PODT varies from 2.462 to 2.889 from several physical models. It was evaluated that the results obtained could contribute to the production of organic electronic devices.

\section{Acknowledgements}

Muş Alparslan University partly supported to this study.

\section{References}

[1] Xin Guo, Martin Baumgarten, Klaus Müllen, 38 (12) (2013) 1832-1908.

[2] https://www.merriam-webster.com/dictionary/reflectance

[3] Mehmet Cabuk, Bayram Gündüz, Colloids and Surfaces A 532 (2017) 263-269.

[4] S.K. Tripathy, Opt. Mater. 46 (2015) 240-246.

[5] Cahit Orek, Bayram Gündüz, Omer Kaygili, Niyazi Bulut, Chemical Physics Letters 678 (2017) 130-138.

[6] Mantarc1, Asim, Gündüz, Bayram, Optical and Quantum Electronics, Vol.48, pp.547, 2016. https://link.springer.com/article/10.1007/s11082-016-0822-5

[7] Mantarc1, Asim, Kundakçi, Mutlu, Bulletin of Materials Science, Vol.42, pp.196, 2019. https://link.springer.com/article/10.1007/s12034-019-1883-4

[8] Mantarci, Asim, Emerging Materials Research, Vol.8 (3), pp.320-330, 2019. https://www.icevirtuallibrary.com/doi/abs/10.1680/jemmr.19.00013

[9] Mantarci, Asim, Mutlu Kundakçi, Materials Science - Poland, Ahead of Print, 2019. https://content.sciendo.com/view/journals/msp/ahead-of-print/article-10.2478-msp-20190052.xml 